\title{
Contribution of horizontal gene transfer to the emergence of VIM-4 carbapenemase producer Enterobacteriaceae in Kuwait
}

This article was published in the following Dove Press journal: Infection and Drug Resistance

\author{
Ágnes Sonnevend' \\ Nour Yahfoufi ${ }^{1,2}$ \\ Akela Ghazawi' \\ Wafaa Jamal ${ }^{3}$ \\ Vincent Rotimi ${ }^{3}$ \\ Tibor Pál'
}

'Department of Medical Microbiology and Immunology, College of

Medicine and Health Sciences,

United Arab Emirates University, Al

Ain, UAE; ' ${ }^{2}$ epartment of Cellular

and Molecular Medicine, Faculty

of Medicine, University of Ottawa,

Ottawa, ON, Canada; ${ }^{3}$ Department of Microbiology, Faculty of Medicine, Kuwait University, Kuwait City, Kuwait
Correspondence: Tibor Pál

Department of Medical Microbiology and Immunology, College of Medicine and Health Sciences, United Arab Emirates University, PO Box 17666, Al Ain, UAE $\mathrm{Tel}+97|37| 37480$

Fax +97I $3767 \mid 966$

Email tpal@uaeu.ac.ae

\begin{abstract}
Carbapenem-resistant Enterobacteriaceae encountered in countries of the Arabian Peninsula usually produce OXA-48-like and New Delhi metallo-beta-lactamases (NDM) carbapenemases. However, a temporary increase in VIM-4-producing, clonally unrelated Enterobacteriaceae strains was described earlier in a Kuwaiti hospital. We investigated the genetic support of bla ${ }_{\mathrm{VIM}-4}$ in six Klebsiella pneumoniae strains, one Escherichia coli, and one Enterobacter cloacae strain and compared it to that of VIM-4-producing isolates from other countries of the region. Five K. pneumoniae strains and the E. coli strain from Kuwait carried an $\sim 165 \mathrm{~kb}$ IncA/C-type plasmid indistinguishable by restriction fragment length polymorphism. The complete sequence of one of them (pKKp4-VIM) was established. pKKp4-VIM exhibited extensive similarities to episomes pKP-Gr642 carrying $b l a_{\mathrm{VIM}-19}$ encountered in Greece and to the partially sequenced pCC416 harboring $b l a_{\mathrm{VIM}-4}$ detected in Italy. In other countries of the region, the only similar plasmid was the one detected in the isolate from the UAE. In all Kuwaiti strains, irrespective of the species and their VIM plasmids, the $b l a_{\mathrm{VIM}-4}$ gene was located within the same integron structure (In416), different from those of other countries of the region. Our data show that the spread of this IncA/C plasmid and particularly that of the In416 integron caused a considerable, albeit temporary, increase in the rate of mostly clonally unrelated VIMproducing Enterobacteriaceae strains of multiple species. Monitoring of such events is of high importance as the interference with the spread of mobile genetic elements may represent a formidable challenge to infection control.
\end{abstract}

Keywords: Enterobacteriaceae, VIM carbapenemase, horizontal gene transfer, multidrug resistance, Middle East

\section{Introduction}

The emergence and spread of carbapenemase-producing Enterobacteriaceae (CPE) is a serious global threat that considerably limits therapeutic options available for lifethreatening Gram-negative infections. Carbapenem-hydrolyzing enzymes have been described in the A, B and D classes of beta-lactamases. ${ }^{1}$ Group B enzymes, i.e., the metallo-beta-lactamases (MBLs), are especially worrisome, as recently introduced beta-lactamase inhibitors have no activity against them. ${ }^{2}$

Although it has been observed that countries of the Arabian Peninsula are burdened by $\mathrm{CPE}$, there are as yet no systematic surveillance-based data regarding the magnitude of the problem. However, studies from the region have shown that locally class D OXA-48-like enzymes and New Delhi metallo-beta-lactamases (NDM) are the most common carbapenemases in Enterobacteriaceae with sporadic occurrence of KPC- 
and VIM-type enzymes. ${ }^{3-7}$ An exception to this trend was a temporarily increased prevalence of VIM-producing strains in Kuwait between 2009 and 2011. Early investigations in Kuwait showed that a few of these strains were clonally related only. ${ }^{8}$ In the current study, we investigate the role of mobile genetic elements in the increased number of VIMpositive isolates in Kuwait and compared the plasmids and integrons to other bla $a_{\mathrm{VIM}}$-bearing mobile genetic elements identified in other countries of the region.

\section{Materials and methods Bacterial strains}

Five Klebsiella pneumoniae (KKp1, KKp2, KKp4, KKp6 and KKp8), one Enterobacter cloacae (KEcl3) and one Escherichia coli (KEc7) were previously described as part of a VIM-producing Enterobacteriaceae outbreak in Kuwait. ${ }^{8} \mathrm{~A}$ further VIM producing K. pneumoniae (KW11) isolated in the same hospital during the same period was also included in the study. The characteristics of these isolates were compared to those of four VIM-producing E. cloacae (two [OM63 and OM69] from Oman, one [SA4/2] from the Kingdom of Saudi Arabia [KSA] and one [ABC104] from the UAE, respectively). ${ }^{4,6}$ All isolates were recovered from individual patients and were considered clinically relevant. The strains were stored at $-80^{\circ} \mathrm{C}$ in Tryptic Soy Broth (Mast, Merseyside, UK) containing $20 \%$ glycerol.

\section{Antibiotic susceptibility assays}

Susceptibility to cefotaxime, ceftazidime, aztreonam, ertapenem, meropenem, imipenem, ciprofloxacin, gentamicin, amikacin, trimethoprim/sulfamethoxazole, tetracycline, chloramphenicol and colistin (Col) was tested by broth microdilution, while susceptibility to fosfomycin (Fos) and tigecycline (Tig) was assessed by agar dilution. ${ }^{9}$ For the majority of antibiotics the Clinical and Laboratory Standards Institute (CLSI) clinical breakpoints were used for interpretation, ${ }^{9}$ with the exception of Col, Tig and Fos whereby the The European Committee on Antimicrobial Susceptibility Testing (EUCAST) criteria. $^{10}$

\section{Molecular typing}

The XbaI-digested genomic DNA's pulsed field gel electrophoresis (PFGE) pattern and the multi-locus sequence type of the isolates were established as described earlier. ${ }^{6,7}$ The Kuwaiti $K$. pneumoniae isolates were also compared by repetitive element sequence-based polymerase chain reaction (rep-PCR; DiversiLab; bioMerieux, Marcy l'Etoile France) using the Klebsiella kit, according to the manufacturer's recommendation. Resistance genes $\left(b l a_{\mathrm{TEM}}, b l a_{\mathrm{CTX}-\mathrm{M}}, b l a_{\mathrm{SHV}}\right.$ bla $a_{\mathrm{PER}}$, bla $a_{\mathrm{AmpC}}, b l a_{\mathrm{NDM}}$, bla $a_{\mathrm{OXA}-48-\mathrm{like}}, b l a_{\mathrm{KPC}}, b l a_{\mathrm{VIM}}, b l a_{\mathrm{IMP}}, a r m A$, $r m t A, r m t B, r m t C, r m t D, r m t E, r m t F, q n r S$, qepA, aac6-1b-cr, $m c r-1, m c r-2)$ were detected as described. ${ }^{4,7,11,12}$ The specific alleles of beta-lactamase genes were determined by direct sequencing of the respective amplicons performed with the BigDye Cycle Terminator V.3.1 (Thermo Fisher Scientific, Waltham, MA, USA) using the 3130X Genetic Analyzer (Thermo Fisher Scientific).

\section{Characterization of the genetic environment of bla $a_{\mathrm{VIM}-4}$}

The flanking region of the $b l a_{\mathrm{VIM}-4}$ gene was determined by polymerase chain reaction (PCR) mapping and sequencing using primers designed (Table S1) according to the genetic surrounding of $b l a_{\mathrm{VIM}}$ published earlier (GenBank accession numbers AJ704863 and AY339625). ${ }^{13,14}$ Sequences were assembled with Clone Manager v9.0 (Sci-Ed Software, Cary, NC, USA) and annotated using Sequin (http://www.ncbi.nlm.

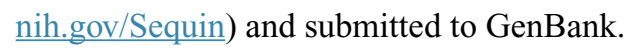

\section{Plasmid characterization}

Plasmids were isolated and detected by the alkaline lysis method as described ${ }^{6}$ using E. coli 39R861 as plasmids molecular size standards. ${ }^{15}$ Mating out assays were performed with the clinical isolates using an azide-resistant derivative of rifampicin-resistant $E$. coli J53 (J53RAZ) as recipient. Transconjugants were selected on Tryptic Soy Agar containing $8 \mathrm{mg} / \mathrm{L}$ ceftazidime and $100 \mathrm{mg} / \mathrm{L}$ azide. ${ }^{7}$ If they were non-conjugative, heat shock transformation of the carbapenemase-bearing plasmids into E. coli DH5 $\alpha$ was attempted. ${ }^{5}$ To prove the loclization of genes, electrophoretically separated plasmids isolated from the wild-type strains by the alkaline lysis method ${ }^{6}$ and those of the transconjugants or transformants were capillary transferred to Hybond $\mathrm{N}+$ membranes that were subsequently hybridized with the appropriate digoxigenin-labeled (Roche Diagnostics $\mathrm{GmbH}$, Mannheim, Germany) probes. ${ }^{6}$ The incompatibility (Inc) groups of the plasmids transferred were identified by $\mathrm{PCR}^{16,17}$ and confirmed by hybridization as mentioned earlier. ${ }^{7}$

Plasmids were purified from single plasmid containing E. coli $\mathrm{K}-12$ derivatives using the Plasmid Maxiprep Kit (Qiagen NV, Venlo, the Netherlands). Restriction patterns of similarly sized plasmids belonging to the same Inc type were visually compared after digesting with HincII, HindIII and EcoRI restriction endonucleases. Furthermore, the complete sequence of pKKp4-VIM conjugally transferred from $K$. pneumoniae KKp4 into E. coli J53RAZ was established 
by next-generation sequencing on Illumina MiSeq platform (performed at the CCIB DNA Core Facility in Massachusetts General Hospital, Cambridge, MA, USA). The gaps between contigs assembled were closed by PCR and by direct sequencing of the amplicons. The complete plasmid sequence was assembled with Clone Manager v9.0, annotated using Sequin (http://www.ncbi.nlm.nih.gov/Sequin) and submitted to GenBank (MF582638).

\section{Ethics approval}

The ethics approval for the study was obtained from the Medical Ethics Committee of the Ministry of Health, Kuwait (288/MTT).

\section{Results}

\section{Antibiotic susceptibility}

The antibiotic susceptibility test results of the isolates are summarized in Table 1, while the respective minimal inhibitory concentration (MIC) values are provided in Table S1. All strains showed resistance to all beta-lactams tested. All Kuwaiti strains were multidrug resistant, with two being susceptible to Col only and one (K. pneumoniae KW11) being resistant to all antibiotics tested (Tables 1 and $\mathrm{S} 2$ ).

\section{Molecular typing}

All strains investigated carried a single carbapenemase, bla $_{\text {VIM-4. }}$ The molecular characteristics of the clinical isolates are summarized in Table 1. Confirming the data of the previous study, i.e., of the Kuwaiti strains, only $K$. pneumoniae KKp1 and KKp2 exhibited similar PFGE patterns (KP-4; Figure S1). ${ }^{8}$ With the exception of these two isolates, the sequence types (Table 1) and the rep-PCR patterns (Figure S2) of the other K. pneumoniae strains were all different. The sole E. cloacae (KEcl3) from Kuwait was different from the other four VIM-4-producing E. cloacae from the region both by PFGE and by multi-locus sequence typing (MLST) (Table 1 and Figure S1).

\section{Comparison of the plasmids carrying bla $a_{\mathrm{VIM}-4}$}

Unlike in the previous study, ${ }^{8}$ we could conjugally transfer VIM-coding plasmids from six of the eight Kuwaiti strains as well as from the Saudi E. cloacae SA4/2. From the Omani E. cloacae isolates (OM63 and OM69), the VIM plasmids were transferred by transformation. From $K$. pneumoniae KW11 and E. cloacae KEcl3 and ABC104, neither conjugations nor transformations were successful.

As confirmed by PCR and by Southern hybridization (Figure S3), in addition to the two clonally related
K. pneumoniae (KKp1 and KKp2), three unrelated K. pneumoniae (KKp4, KKp6 and KKp8) isolates and the E. coli (KEc7) from Kuwait harbored bla $a_{\mathrm{VIM}-4}$ on IncA/C Inc-type plasmids of $\sim 165 \mathrm{~kb}$. Beyond bla $a_{\mathrm{VIM}-4}$, these plasmids also carried the $b l a_{\text {CMY-4 }}$ gene (Table 1). The RFLP patterns of these plasmids were identical (Figure 1). E. cloacae ABC104 from the UAE (described earlier ${ }^{6}$ ) also carried $b l a_{\mathrm{VIM}-4}$ and $b l a_{\mathrm{CMY}-4}$ on similar-sized IncA/C Inc-type plasmid, but we were unable to compare the RFLP of this plasmid to the Kuwaiti ones as we could not generate a single VIM plasmid-containing derivative of this isolate. In $K$. pneumoniae $\mathrm{KW} 11$, the bla $_{\mathrm{VIM}-4}$ was located on a nontransferable IncA/C type, $>300 \mathrm{~kb}$ plasmid, which also carried $b l a_{\mathrm{CMY}-4}$. As shown in Table 1 and in Figure S3, the Kuwaiti, Saudi and Omani E. cloacae isolates all carried $b l a_{\mathrm{VIM}-4}$ on smaller plasmids lacking $b l a_{\mathrm{CMY}-4}$, which could not be identified by the PCR-based replicon typing (PBRT). In case of KKp1 and KKp2, the conjugal transfer of the bla ${ }_{\mathrm{VIM}}$-bearing plasmids was accompanied by their fusion with IncN-type plasmids (Figure S4). No attempts were made, within the frames of the current study, to clarify the molecular details of this fusion.

\section{Complete sequence of pKKp4-VIM}

To obtain a more detailed picture on the conjugative IncA/Ctype plasmids dominating the isolates from Kuwait, the entire sequence was determined from a transconjugant containing pKKp4-VIM derived from $K$. pneumoniae KKp4. The plasmid was a $162117 \mathrm{bp}$ long, type $1 \mathrm{IncA} / \mathrm{C}_{2}$ plasmid with respective regions for replication, conjugative transfer and plasmid maintenance (GenBank Accession No. MF582638). It was highly similar to $\mathrm{pKP}-\mathrm{Gr} 642$, a type $1 \mathrm{IncA} / \mathrm{C}_{2}$-type plasmid of a Greek clinical $K$. pneumoniae isolate carrying bla ${ }_{\mathrm{VIM}-19}\left(\right.$ Figure 2A) ${ }^{18}$. Apart from the plasmid backbone, pKKp4-VIM harbored three resistance islands: RI-1, RI-2 and RI-3 (Figure 2A). On RI-1 tet(A), strA, strB and sul2 genes are located (Figure 2B). The RI-2 consists of ISEcpl, bla ${ }_{\mathrm{CMY}-4}$, blc and $\operatorname{sug} E$ genes. The third resistance island RI-3 contains an In 416 with $b l a_{\mathrm{VIM}-4,} a a c A 7, d f r A 1, \Delta a a d A 1$ and smr gene cassettes, a Tn 8802 with arsenic resistance operon, an In-t4-like integron and a mercury resistance operon (Figure 2D).

\section{Genetic surrounding of bla $a_{\mathrm{VIM}-4}$ in all isolates}

PCR mapping and sequencing revealed that irrespective of the species or plasmid Inc type, the integron structure of all Kuwaiti isolates was identical to the one in pKKp4-VIM, i.e., bla $_{\mathrm{VIM}-4}$ was located on an In416 integron, which lacked the $3^{\prime}$ conserved sequences (CS). On the other hand, in E. cloacae 


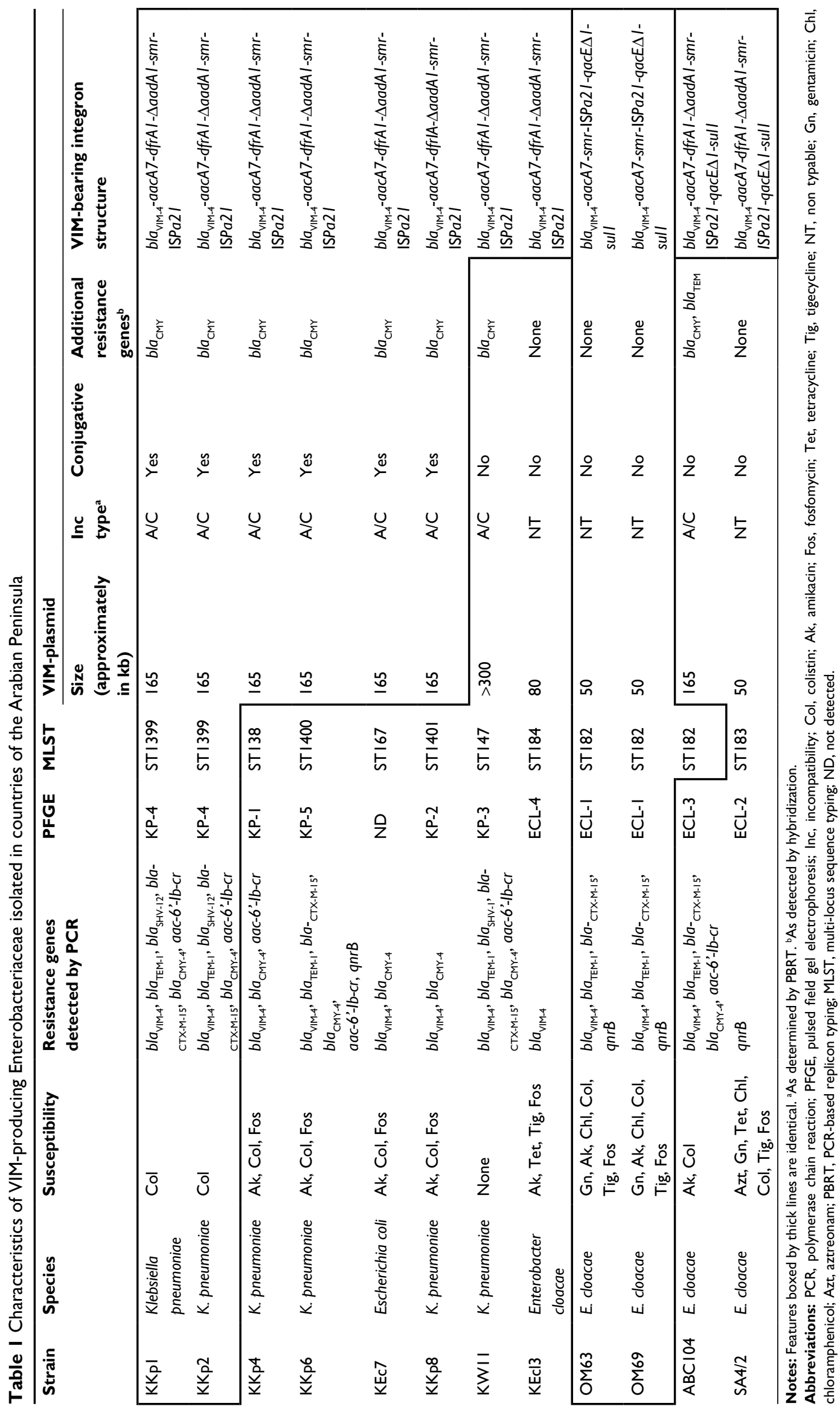




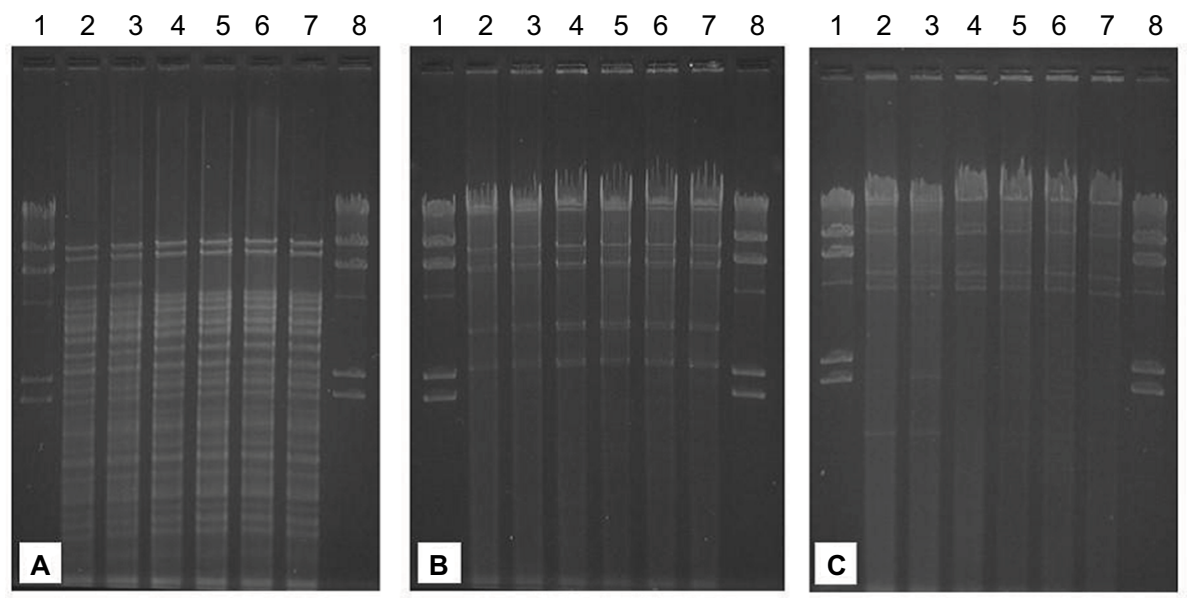

Figure I Restriction fragment length polymorphism of conjugative IncA/C plasmids of the Kuwaiti isolates.

Notes: (A) Digestion with Hincll. (B) Digestion with EcoRI. (C) Digestion with HindlII. Lanes I and 8, lambda phage DNA digested with Hindlll; Lane 2, pKKp I-VIM; Lane 3, pKKp2-VIM; Lane 4, pKKp4-VIM; Lane 5, pKKp6-VIM; Lane 6, pKEc7-VIM and Lane 7, pKKp8-VIM.

ABC104 described earlier from the UAE (GenBank Accession No. JX275775) ${ }^{6}$ and in E. cloacae SA4/2 from Saudi Arabia, the qacE 11 -sul1-orf5 structure was present downstream of the ISPa21. In the two Omani isolates, the integron lacked the $d f r A 1$ and $\triangle a a d A 1$ cassettes, and the $3^{\prime}$ CS was present downstream of ISPa21 (GenBank Accession No. MF178139; Table 1 and Figure 2C).

\section{Discussion}

VIM-producing Enterobacteriaceae have only been sporadically encountered in countries of the Arabian Peninsula. ${ }^{4,6,19,20}$ Between April 2009 and February 2011, a higher prevalence of mostly unrelated VIM-4 producer Enterobacteriaceae strains was observed in Kuwait. ${ }^{8}$ As shown by our current data, this increased rate of $b l a_{\mathrm{VIM}}$-carrying strains was mostly due to local horizontal gene transmission, leading to the uniform presence of the same $b l a_{\mathrm{VIM}}$-containing In416 integron in all Kuwaiti isolates, irrespective of the species and plasmids carried, and to the wide distribution of a $162 \mathrm{~kb}$ IncA/ $\mathrm{C}_{2}$-type plasmid. It is noteworthy that this type of integron and plasmid was characteristic to the Kuwaiti isolates, whereas in strains from other countries of the region, there was much heterogeneity of episomes and integrons (Table 1 and Figure 2C).

It is of interest that a plasmid (pKP-Gr642) very similar to the one spreading bla ${ }_{\mathrm{VIM}-4}$ in Kuwait was found in Greece. It bore a single amino acid variant of this enzyme, i.e., bla $a_{\mathrm{VIM}-19}{ }^{18}$ The slight differences between this latter plasmid and pKKp4-VIM are highlighted in Figure 2. The RI-1 of pKP-Gr642 contains an additional ISCR2-driven floR gene (Figure 2B), and its RI-3 lacks the In-t4-like integron containing $a a d B, c m l A 7$, qacE $\Delta 1$ and sull genes (Figure 2D).
Furthermore, pKP-Gr642 carries two insertion elements (ISEc23 and ISVpa4) in the plasmid backbone.

Moreover, features of pCC416, the conjugative IncA/C plasmid shown to transfer bla $a_{\mathrm{VIM}-4}$ between E. cloacae and $K$. pneumoniae clinical isolates of a patient in Italy, ${ }^{14,21}$ were closely similar to the endemic pKKp4-VIM of Kuwait. Both RI-2 and RI-3 of pKKp4-VIM were 99\% identical to the two fragments sequenced of pCC416 (GenBank Accession Nos. AJ875405 and AJ704863). Furthermore, pCC416 was also reported to carry a sul2 gene, which is located on RI-1 of pKKp4-VIM. After detecting its in vivo transfer in a patient, it was speculated that this particular IncA/C plasmid could play a role in the spread of carbapenem resistance. ${ }^{14,21}$ Our study confirmed this hypothesis by showing that such plasmids are indeed able to spread, over a year-long period, between strains carried by different patients, not even respecting species' barriers.

A limitation of our study is the lack of epidemiological data linking the cases to each other. No details were available to us regarding possible routes of transmission. Furthermore, only eight strains (seven of the originally described outbreak set of 11 plus one further isolate) were available for the investigation. However, even the data on this set of strains showed the heterogeneity of strains being in sharp contrast with the near uniformity of plasmids and the complete identity of $b l a_{\text {VIM-4 }}$ containing integrons in all Kuwaiti isolates. Importantly, this increased rate of VIM-positive isolates seems to be a temporary event, as strains collected subsequently from Kuwait and even from the same hospital expressed mostly NDM- and OXA-type enzymes, otherwise characteristic of the region. ${ }^{4,22}$ 
A

pKKp4-VIM 162117 bp MF582638

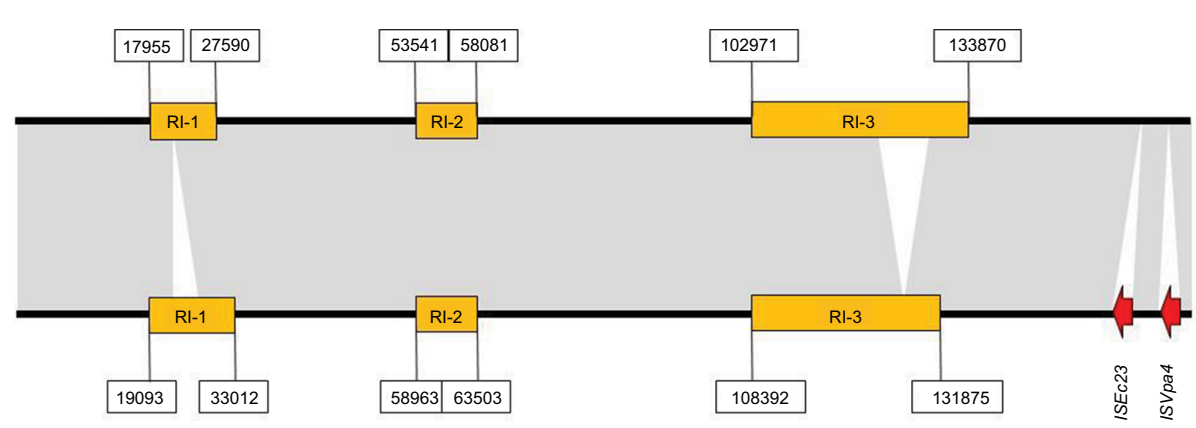

B pKP-Gr642 162787 bp KR559888

RI-1 of pKKp4-VIM

Rl-1 of pKP-Gr642

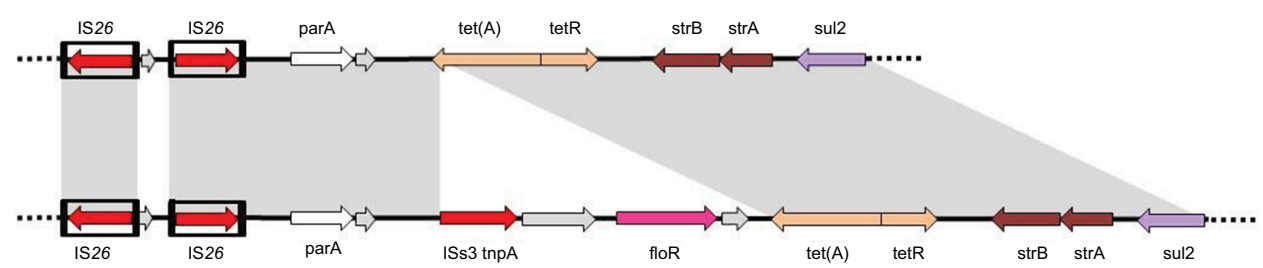

C

Kuwaiti VIM integron (In416)

UAE and Saudi VIM integron

Omani VIM integron

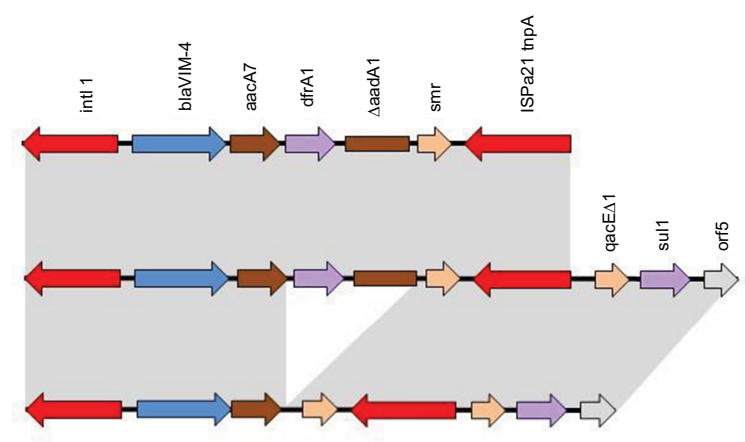

D

RI-3 of pKKp4-VIM

RI-3 of pKP-Gr642

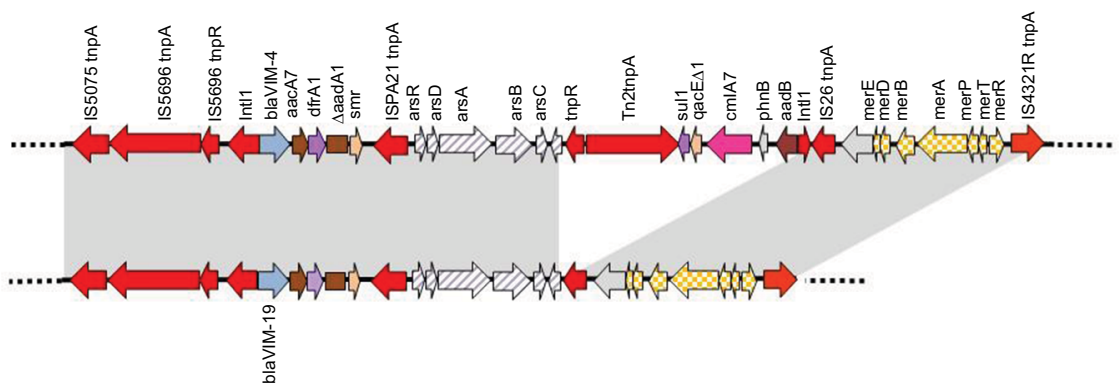

Figure 2 Structure of pKKp4-VIM.

Notes: (A) Comparison of the complete pKKp4-VIM to pKP-Gr642. (B) Comparison of RI-I of pKKp4-VIM and pKP-Gr642. (C) Comparison of the three bla VIM-4. bearing integron variants. (D) Comparison of RI-3 of pKKp4-VIM to RI-3 of pKP-Gr642. Gray areas represent $\geq 95 \%$ similarity.

Our results also highlight the importance of the detailed molecular typing of CPE to obtain a realistic picture of the complexity of the spread of carbapenem resistance. The spread of plasmids and integrons represents a considerable challenge to infection control. Horizontal gene transfer is difficult to prevent by routine infection control measures, and only limiting the selective antibiotic pressure in the human body and in the environment may possibly mitigate its efficacy.

\section{Acknowledgments}

The kind help of Ms Geraldine Kershaw (CMHS, UAE University) in language editing the manuscript is highly 
appreciated. This work was supported by grants UPAR25143 (31R061) and UAEU FMHS grants NP/12/13 and NP-10-11/1019 awarded to TP and UAEU UPAR-31M235 grant awarded to AS.

\section{Disclosure}

The authors report no conflicts of interest in this work.

\section{References}

1. Diene SM, Rolain JM. Carbapenemase genes and genetic platforms in Gram-negative bacilli: Enterobacteriaceae, Pseudomonas and Acinetobacter species. Clin Microbiol Infect. 2014;20(9):831-838.

2. Falcone M, Paterson D. Spotlight on ceftazidime/avibactam: a new option for MDR Gram-negative infections. J Antimicrob Chemother. 2016;71(10):2713-2722.

3. Zowawi HM, Sartor AL, Balkhy HH, et al. Molecular characterization of carbapenemase-producing Escherichia coli and Klebsiella pneumoniae in the countries of the Gulf cooperation council: dominance of OXA-48 and NDM producers. Antimicrob Agents Chemother. 2014;58(6):3085-3090.

4. Sonnevend A, Ghazawi AA, Hashmey R, et al. Characterization of carbapenem-resistant Enterobacteriaceae with high rate of autochthonous transmission in the Arabian Peninsula. PLoS One. 2015;10(6):e0131372.

5. Sonnevend A, Ghazawi A, Darwish D, AlDeesi Z, Kadhum AF, Pal T. Characterization of KPC-type carbapenemase-producing Klebsiella pneumoniae strains isolated in the Arabian Peninsula. J Antimicrob Chemother. 2015;70(5):1592-1593.

6. Sonnevend A, Ghazawi A, Yahfoufi N, et al. VIM-4 carbapenemaseproducing Enterobacter cloacae in the United Arab Emirates. Clin Microbiol Infect. 2012;18(12):E494-E496.

7. Sonnevend A, Al Baloushi A, Ghazawi A, et al. Emergence and spread of NDM-1 producer Enterobacteriaceae with contribution of IncX3 plasmids in the United Arab Emirates. J Med Microbiol. 2013;62(pt 7):1044-1050.

8. Jamal W, Rotimi VO, Albert MJ, Khodakhast F, Nordmann P, Poirel L. High prevalence of VIM-4 and NDM-1 metallo-beta-lactamase among carbapenem-resistant Enterobacteriaceae.J Med Microbiol. 2013;62(pt 8): 1239-1244.

9. CLSI. Performance Standard for Antimicrobial Susceptibility Testing; M100-S24. Wayne, PA: Clinical and Laboratory Standard Institute; 2014.
10. EUCAST. European Committee on Antimicrobial Susceptibility Testing. [homepage on the Internet]. http://www.eucast.org/. Accessed November 10, 2017

11. Liu YY, Wang Y, Walsh TR, et al. Emergence of plasmid-mediated colistin resistance mechanism MCR-1 in animals and human beings in China: a microbiological and molecular biological study. Lancet Infect Dis. 2016;16(2):161-168.

12. Xavier BB, Lammens $C$, Ruhal $R$, et al. Identification of a novel plasmidmediated colistin-resistance gene, mcr-2, in Escherichia coli, Belgium, June 2016. Euro Surveill. 2016;21(27).

13. Miriagou V, Tzouvelekis LS, Villa L, et al. CMY-13, a novel inducible cephalosporinase encoded by an Escherichia coli plasmid. Antimicrob Agents Chemother. 2004;48(8):3172-3174.

14. Colinon C, Miriagou V, Carattoli A, Luzzaro F, Rossolini GM. Characterization of the IncA/C plasmid pCC416 encoding VIM-4 and CMY-4 beta-lactamases. J Antimicrob Chemother. 2007;60(2):258-262.

15. Macrina FL, Kopecko DJ, Jones KR, Ayers DJ, McCowen SM. A multiple plasmid-containing Escherichia coli strain: convenient source of size reference plasmid molecules. Plasmid. 1978;1(3):417-420.

16. Carattoli A, Bertini A, Villa L, Falbo V, Hopkins KL, Threlfall EJ. Identification of plasmids by PCR-based replicon typing. J Microbiol Methods. 2005;63(3):219-228.

17. Johnson TJ, Bielak EM, Fortini D, et al. Expansion of the IncX plasmid family for improved identification and typing of novel plasmids in drugresistant Enterobacteriaceae. Plasmid. 2012;68(1):43-50.

18. Papagiannitsis CC, Dolejska M, Izdebski R, et al. Characterisation of IncA/C2 plasmids carrying an In416-like integron with the blaVIM-19 gene from Klebsiella pneumoniae ST383 of Greek origin. Int J Antimicrob Agents. 2016;47(2):158-162.

19. Yezli S, Shibl AM, Memish ZA. The molecular basis of beta-lactamase production in Gram-negative bacteria from Saudi Arabia. J Med Microbiol. 2015;64(pt 2):127-136.

20. Memish ZA, Assiri A, Almasri M, et al. Molecular characterization of carbapenemase production among Gram-negative bacteria in Saudi Arabia. Microb Drug Resist. 2015;21(3):307-314.

21. Luzzaro F, Docquier JD, Colinon C, et al. Emergence in Klebsiella pneumoniae and Enterobacter cloacae clinical isolates of the VIM-4 metallo-beta-lactamase encoded by a conjugative plasmid. Antimicrob Agents Chemother. 2004;48(2):648-650.

22. Jamal WY, Albert MJ, Rotimi VO. High prevalence of New Delhi metallo-beta-lactamase-1 (NDM-1) producers among carbapenem-resistant Enterobacteriaceae in Kuwait. PLoS One. 2016;11(3):e0152638. 


\section{Supplementary materials}

Table SI Primers used in sequencing the molecular structures carrying the bla $a_{\mathrm{VIM}}$ gene

\begin{tabular}{|c|c|c|c|c|}
\hline Primer name & $5^{\prime}-3^{\prime}$ sequence & $\begin{array}{l}\text { Annealing to } \\
\text { AJ704863 }\end{array}$ & $\begin{array}{l}\text { Size of } \\
\text { products (bp) }\end{array}$ & Comment \\
\hline AS_Classlint_L & TGT CGT TTT CAG AAG ACG GCT GC & 5250 & 434 & For amplification and sequencing the $5^{\prime}$ \\
\hline AS_Classlint_R & CAA ACG TGC CGT AGA ACA AG & $5683 C$ & & end of class I integron \\
\hline AS_intlI_L & GGG AGG ACT TTC CGC AAC CG & 5363 & 1084 & For amplification and sequencing the \\
\hline AS_VIM_R & CGT TAC CAC CGC TGC GTT CG & $6446 C$ & & $b l a_{\mathrm{vIM}}$ upstream region \\
\hline AS_VIM_GS_SI & GCC TTG ATG TTA CCC GAG AG & $5937 C$ & NA & \\
\hline AS-VIM4GS-f & GAT GCG TGG AGA CCG AAA CC & 6228 & 1295 & For amplification and sequencing the \\
\hline AS-VIM4GS-r & TGC CTA ACG CCT GAG TTG AG & $7522 C$ & & $b l a_{\mathrm{VIM}}$ and its immediate surroundings \\
\hline AS_VIM_L & AAT CGC TCA GTC GCC GAG TA & 7412 & 3750 & For amplification and sequencing the \\
\hline AS_ISPa2I_R & CTA TAA GAC ACG AGG TGT CTG & $1116 I C$ & & $b l a_{\mathrm{VIM}}$ downstream region \\
\hline AS_ISPa2I_L & CAC CAC AAC CGC AAG AAA TA & 10034 & NA & \\
\hline AS_ISPa2I_seq & CGC GCA TCG ATT GTT CGT AG & 10549 & NA & \\
\hline AS_smr_f & GCT GGA CTC TTT GAG ATT GG & 9507 & NA & \\
\hline AS_dhfrl_R & ACC CTT TTG CCA GAT TTG GT & $8597 C$ & NA & \\
\hline AS_aacA7_R & GAG CAA CCT CCG TGA ATC CA & $7955 C$ & NA & \\
\hline AS_VIMdn_LSI & TTC GTT CAA GCC GAA CTT GC & 8010 & NA & \\
\hline AS_VIMdn_LS2 & AAT AGA CAT CGA GCC GGA AG & 8477 & NA & \\
\hline AS_VIMdn_LS3 & ACA TAG CGT TGC CTT GGT AG & 9030 & NA & \\
\hline AS_orf5_R & TTA GAT TTC GAG TTC TAG GCG TTC TG & NA & 3647 bp (if & Primer AS_orf5_R anneals to the $3^{\prime}$ end \\
\hline AS_smr_f & GCT GGA CTC TTT GAG ATT GG & 9507 & $\begin{array}{l}\text { class I integron } \\
\text { classical } 3^{\prime} \text { end is } \\
\text { present) }\end{array}$ & $\begin{array}{l}\text { of the class I integron; the two primers } \\
\text { amplify the } 3^{\prime} \text { region of class I integron } \\
\text { if present }\end{array}$ \\
\hline AS_ISPa2I_R & CTA TAA GAC ACG AGG TGT CTG & $11161 \mathrm{C}$ & NA & $\begin{array}{l}\text { Sequencing the amplicon produced by } \\
\text { the PCR above }\end{array}$ \\
\hline AS_ISPa2I_L & CAC CAC AAC CGC AAG AAA TA & 10034 & NA & \\
\hline AS_ISPa2I_seq & CGC GCA TCG ATT GTT CGT AG & 10549 & NA & \\
\hline AS_sulI_RI & TTG CCG ATC GCG TGA AGT TC & $13000 \mathrm{C}$ & NA & $\begin{array}{l}\text { Sequencing the amplicon produced by } \\
\text { the PCR using primer AS_orf5_R and } \\
\text { AS_smr_f }\end{array}$ \\
\hline AS_sull_R2 & CACAACCTGGTCGATATCAC & I33IIC & NA & \\
\hline AS_orf5_L & ATGGACAGCGAGGAGC & 13249 & NA & \\
\hline AS_qacEDI_L & GCG AAG TAA TCG CAA CAT CC & 11978 & NA & \\
\hline AS_VIMdn_LS4 & GAT CAG ATG CAC CGT GTT TC & $1250 \mid$ & NA & \\
\hline
\end{tabular}

Abbreviations: NA, not applicable; PCR, polymerase chain reaction.

Klebsiella pneumoniae

Dice (Tol 1.5\%-1.5\%) ( $\mathrm{H}>0.0 \% \mathrm{~S}>0.0 \%$ ) [0.0\%-100.0\%]

PFGE

$$
\text { PFGE }
$$
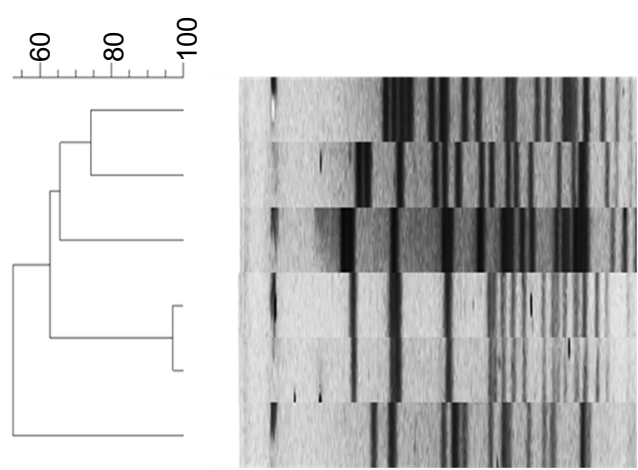

KKp4

KKp8

KW11

KKp1

KKp2

KKp6
Enterobacter cloacae

Dice (Tol 1.5\%-1.5\%) (H>0.0\% S >0.0\%) [0.0\%-100.0\%]

PFGE PFGE

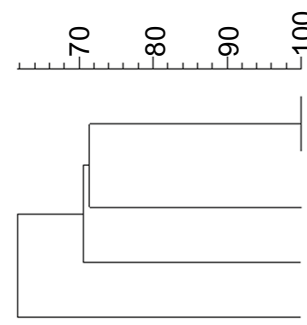

OM63

OM69

$\mathrm{SA} 4 / 2$

$\mathrm{ABC} 104$

$\mathrm{KECl} 3$

Figure SI PFGE comparison of VIM-producing Enterobacteriaceae. Abbreviation: PFGE, pulsed field gel electrophoresis. 
Table S2 MIC values of antibiotics against VIM-producing strains and their derivatives

\begin{tabular}{|c|c|c|c|c|c|c|c|c|c|c|c|c|c|c|c|c|}
\hline Strain & 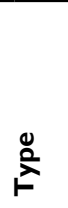 & U⿺ & 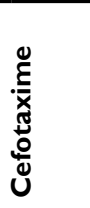 & 嵅 & 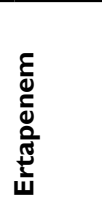 & 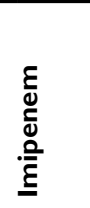 & $\begin{array}{l}\frac{E}{0} \\
\frac{D}{0} \\
\frac{0}{0} \\
\frac{1}{\delta}\end{array}$ & 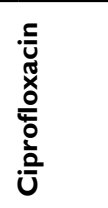 & ن & 这 & 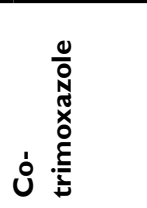 & 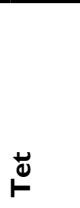 & $\overline{\mathrm{U}}$ & $\bar{ن}$ & $\stackrel{000}{F}$ & ஜ̆ \\
\hline KKpl & W & $>128$ & $>128$ & $>128$ & $>64$ & 128 & 128 & 2 & 256 & 32 & $>256 / 4864$ & $>256$ & $>256$ & $<0.5$ & 2 & 64 \\
\hline J53RAZ(pKKpI-VIM) & $\mathrm{TC}$ & $>128$ & 128 & 128 & 16 & 8 & 2 & $<0.125$ & 32 & 8 & $128 / 2432$ & 32 & 256 & $<0.5$ & 0.25 & 0.5 \\
\hline KKp2 & W & $>128$ & $>128$ & $>128$ & $>64$ & 128 & 128 & 2 & 256 & 32 & $>256 / 4864$ & $>256$ & $>256$ & $<0.5$ & 2 & 64 \\
\hline J53RAZ(pKKp2-VIM) & $\mathrm{TC}$ & 128 & 128 & 128 & 4 & 2 & 2 & $<0.125$ & 32 & 8 & $128 / 2432$ & 32 & 256 & $<0.5$ & 0.25 & 0.5 \\
\hline KEcl3 & W & $>128$ & $>128$ & $>128$ & $>64$ & 64 & 32 & 2 & $>256$ & 16 & $>256 / 4864$ & 4 & 16 & 4 & 0.5 & 32 \\
\hline KKp4 & W & 128 & 128 & 32 & 64 & 16 & 16 & 0.5 & 64 & 16 & $>256 / 4864$ & $>256$ & 256 & $<0.5$ & 8 & 16 \\
\hline J53RAZ(pKKp4-VIM) & $\mathrm{TC}$ & 64 & 32 & 32 & 4 & I & $<0.25$ & $<0.125$ & 16 & 4 & $128 / 2432$ & 32 & 16 & $<0.5$ & 0.25 & 2 \\
\hline KKp6 & W & 128 & $>128$ & $>128$ & 64 & 16 & 8 & 16 & 128 & 8 & $>256 / 4864$ & $>256$ & $>256$ & $<0.5$ & 8 & 4 \\
\hline J53RAZ(pKKp6-VIM) & $\mathrm{TC}$ & 128 & 64 & 64 & 4 & 2 & I & $<0.125$ & 16 & 8 & $128 / 2432$ & 64 & 128 & $<0.5$ & 0.25 & 2 \\
\hline KEc7 & W & $>128$ & $>128$ & 64 & 64 & 8 & 8 & $>64$ & 64 & 16 & $>256 / 4864$ & 128 & 256 & $<0.5$ & 0.5 & 0.5 \\
\hline J53RAZ(pKEc7-VIM) & $\mathrm{TC}$ & 64 & 64 & 64 & 16 & 2 & 0.5 & $<0.125$ & 16 & 8 & $256 / 4864$ & 128 & 256 & $<0.5$ & 0.25 & I \\
\hline ККр8 & W & $>128$ & $>128$ & $>128$ & 64 & 16 & 8 & $>64$ & 128 & 16 & $>256 / 4864$ & $>256$ & $>256$ & $<0.5$ & 0.5 & 16 \\
\hline J53RAZ(pKKp8-VIM) & $\mathrm{TC}$ & 64 & 64 & 32 & 4 & 2 & $<0.25$ & $<0.125$ & 16 & 8 & $128 / 2432$ & 64 & 128 & $<0.5$ & 0.25 & 2 \\
\hline KWII & W & $>128$ & $>128$ & $>128$ & $>64$ & $>128$ & 128 & $>64$ & 256 & 32 & $>256 / 4864$ & $>256$ & 16 & 64 & 2 & 128 \\
\hline $\mathrm{SA} 4 / 2$ & W & 64 & $>128$ & I & 64 & 64 & 16 & I & 2 & 32 & $>256 / 4864$ & 4 & 8 & $<0.5$ & I & 4 \\
\hline J53RAZ(pSA4/2-VIM) & $\mathrm{TC}$ & 32 & 64 & 0.5 & 32 & 4 & 2 & 0.25 & 1 & 8 & $<0.5 / 9.5$ & 2 & 8 & $<0.5$ & $<0.125$ & I \\
\hline OM63 & W & 128 & $>128$ & $>128$ & 32 & 4 & 4 & 64 & 2 & 16 & $>256 / 4864$ & $>256$ & 8 & $<0.5$ & 0.5 & 32 \\
\hline $\mathrm{DH} 5 \alpha(\mathrm{pOM} 63-\mathrm{VIM})$ & $\mathrm{TF}$ & 16 & 32 & $<0.25$ & 2 & 0.5 & $<0.25$ & $<0.125$ & 1 & 8 & $<0.5 / 9.5$ & $<0.5$ & I & $<0.5$ & $<0.125$ & $<0.25$ \\
\hline OM69 & W & 128 & $>128$ & 128 & 64 & 64 & 4 & 32 & 2 & 16 & $>256 / 4864$ & 256 & 8 & $<0.5$ & 0.5 & 32 \\
\hline $\mathrm{DH} 5 \alpha(\mathrm{pOM} 69-\mathrm{VIM})$ & $\mathrm{TF}$ & 16 & 32 & $<0.25$ & 2 & 0.5 & $<0.25$ & $<0.125$ & 1 & 8 & $<0.5 / 9.5$ & $<0.5$ & I & $<0.5$ & $<0.125$ & $<0.25$ \\
\hline $\mathrm{ABClO4}$ & W & $>128$ & $>128$ & $>128$ & $>64$ & 32 & 4 & 32 & 64 & 8 & $>256 / 4864$ & $>256$ & 128 & $<0.5$ & 8 & 64 \\
\hline J53RAZ & $\mathrm{R}$ & $<0.25$ & $<0.25$ & $<0.25$ & $<0.125$ & $<0.25$ & $<0.25$ & $<0.125$ & I & $<0.5$ & $<0.5 / 9.5$ & $<0.5$ & 8 & $<0.5$ & $<0.125$ & 0.5 \\
\hline $\mathrm{DH} 5 \alpha$ & $\mathrm{R}$ & $<0.25$ & $<0.25$ & $<0.25$ & $<0.125$ & $<0.25$ & $<0.25$ & $<0.125$ & I & I & $<0.5 / 9.5$ & $<0.5$ & I & $<0.5$ & $<0.125$ & $<0.25$ \\
\hline
\end{tabular}

Abbreviations: MIC, minimal inhibitory concentration; Azt, aztreonam; Gn, gentamicin; Ak, amikacin; Tet, tetracycline; Chl, chloramphenicol; Col, colistin; Tig, tigecycline; Fos, fosfomycin; W, wild; TC, transconjugant; TF, transformant; R, recipient.

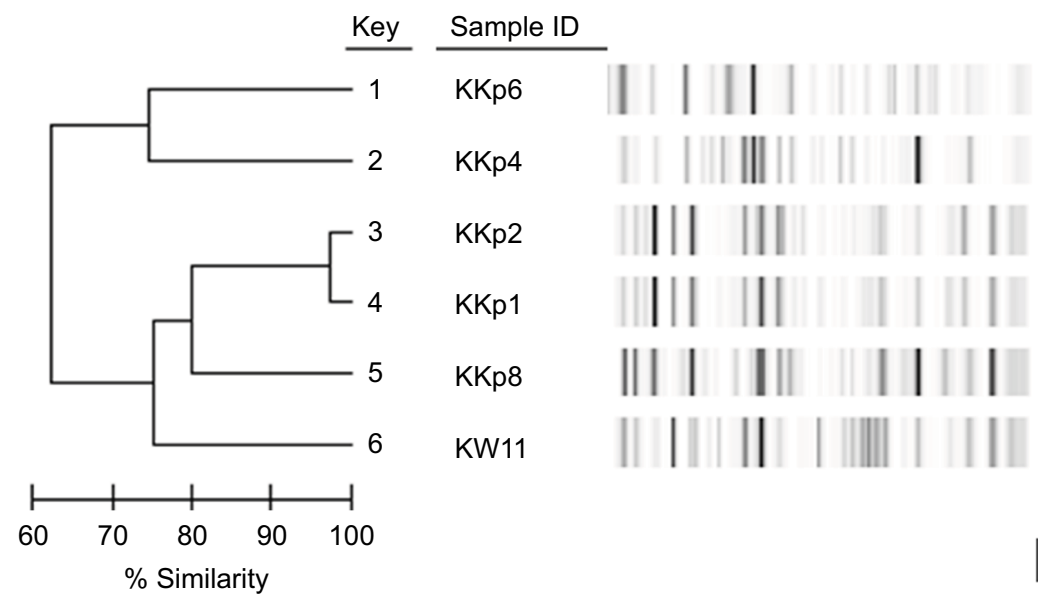

Figure S2 rep-PCR comparison of Kuwaiti Klebsiella pneumoniae strains.

Abbreviation: rep-PCR, repetitive element sequence-based polymerase chain reaction. 

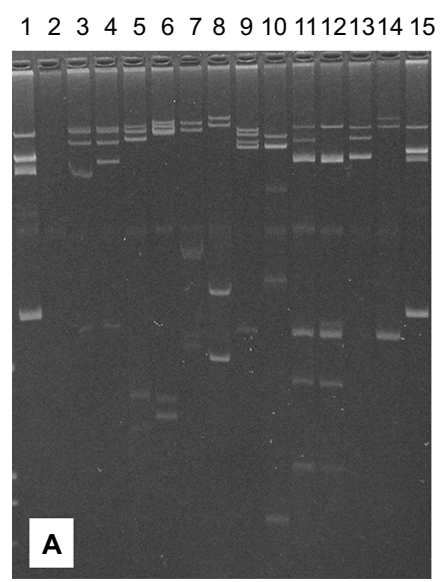

34567891011121314

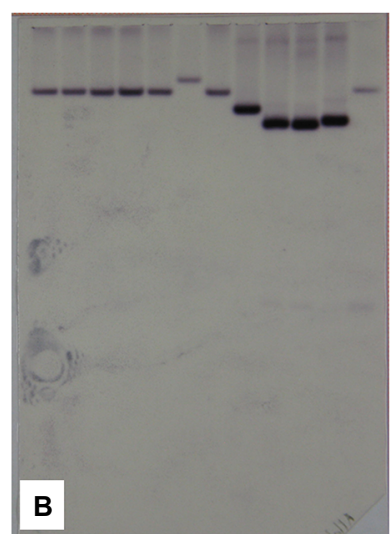

34567891011121314

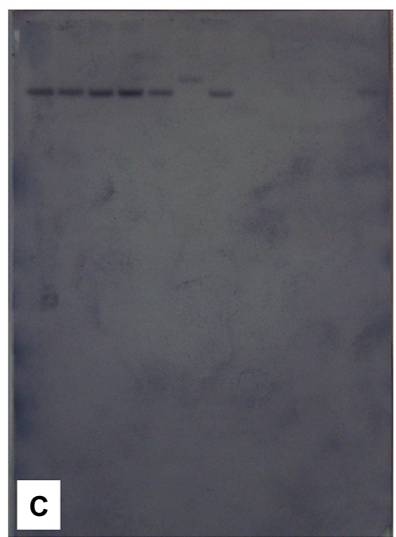

34567891011121314

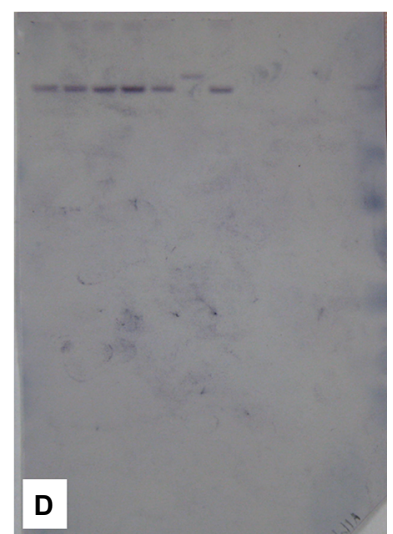

Figure S3 Plasmid profiles of VIM-producing Enterobacteriaceae.

Notes: (A) Plasmid gel. (B) Membrane hybridized with bla $a_{\text {VIM-4 }}$ probe. (C) Membrane hybridized with bla $a_{\mathrm{CMY}-4}$ probe. (D) Membrane hybridized with IncA/C probe. Lane I, Escherichia coli 39R86I; Lane 2, E. coli J53RAZ; Lane 3, Klebsiella pneumoniae KKpl; Lane 4, K. pneumoniae KKp2; Lane 5, K. pneumoniae KKp4; Lane 6, K. pneumoniae KKp6; Lane 7, K. pneumoniae KKp8; Lane 8, K. pneumoniae KWI I; Lane 9, E. coli KEc7; Lane 10, Enterobacter cloacae KEcl3; Lane II, E. cloacae OM63; Lane I2, E. cloacae OM69; Lane I3, E. cloacae SA4/2; Lane I4, E. cloacae ABCI04; Lane I5, E. coli 39R86I.

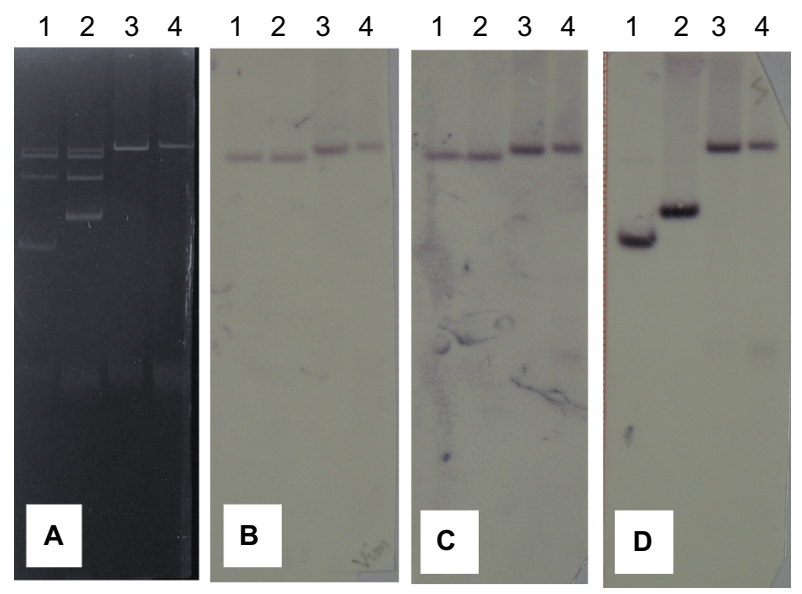

Figure S4 Fusion of IncA/C-VIM and IncN plasmids.

Notes: (A) Plasmid gel. (B) Membrane hybridized with VIM probe. (C) Membrane hybridized with Inc A/C probe. (D) Membrane hybridized with Inc N probe. Lane I, Klebsiella pneumoniae KKpl; Lane 2, K. pneumoniae KKp2; Lane 3, Escherichia coli J53RAZ(pKKpI-VIM); Lane 4, E. coli J53RAZ(pKKp2-VIM).

\section{Publish your work in this journal}

Infection and Drug Resistance is an international, peer-reviewed openaccess journal that focuses on the optimal treatment of infection (bacterial, fungal and viral) and the development and institution of preventive strategies to minimize the development and spread of resistance. The journal is specifically concerned with the epidemiology of antibiotic

\section{Dovepress}

resistance and the mechanisms of resistance development and diffusion in both hospitals and the community. The manuscript management system is completely online and includes a very quick and fair peerreview system, which is all easy to use. Visit http://www.dovepress.com/ testimonials.php to read real quotes from published authors. 